\title{
OPEN Toolbox for the structure-guided evolution of ferulic acid decarboxylase (FDC)
}

\author{
Horia Duță1,2, Alina Filip ${ }^{1,2}$, Levente Csaba Nagy¹, Emma Zsófia Aletta Nagy, \\ Róbert Tőtős $^{1}$ \& László Csaba Bencze ${ }^{1 凶}$
}

The interest towards ferulic acid decarboxylase (FDC), piqued by the enzyme's unique 1,3-dipolar cycloaddition mechanism and its atypic prFMN cofactor, provided several applications of the FDC mediated decarboxylations, such as the synthesis of styrenes, or its diverse derivatives, including 1,3-butadiene and the enzymatic activation of $\mathrm{C}-\mathrm{H}$ bonds through the reverse carboligation reactions. While rational design-based protein engineering was successfully employed for tailoring FDC towards diverse substrates of interest, the lack of high-throughput FDC-activity assay hinders its directed evolution-based protein engineering. Herein we report a toolbox, useful for the directed evolution based and/or structure-guided protein engineering of FDC, which was validated representatively on the well described FDC, originary from Saccharomyces cerevisiae (ScFDC). Accordingly, the developed fluorescent plate-assay allows in premiere the FDC-activity screens of a mutant library in a highthroughput manner. Moreover, using the plate-assay for the activity screens of a rationally designed 23-membered ScFDC variant library against a substrate panel comprising of 16, diversely substituted cinnamic acids, revealed several variants of improved activity. The superior catalytic properties of the hits revealed by the plate-assay, were also supported by the conversion values from their analytical scale biotransformations. The computational results further endorsed the experimental findings, showing inactive binding poses of several non-transformed substrate analogues within the active site of the wild-type ScFDC, but favorable ones within the catalytic site of the variants of improved activity. The results highlight several 'hot-spot' residues involved in substrate specificity modulation of FDC, such as I189, 1330, F397, 1398 or 0192 , of which mutations to sterically less demanding residues increased the volume of the active site, thus facilitated proper binding and increased conversions of diverse non-natural substrates. Upon revealing which mutations improve the FDC activity towards specific substrate analogues, we also provide key for the rational substrate-tailoring of FDC.

Ferulic acid decarboxylase (FDC), as member of the carboxy-lyase family (EC 4.1.1.) has attracted significant interest in recent years, mainly due to its unusual, prenylated flavin mononucleotide (prFMN) cofactor $^{1}$, as well as to its unique mechanism, representing the first enzymatic 1,3-dipolar cycloaddition ${ }^{2,3}$.

Besides the studies focusing on the elucidation of the structure and mechanism of FDC, as first application of FDC, the biosynthesis of styrene, involving the decarboxylation of cinnamic acid within engineered Escherichia coli cells, has been reported ${ }^{4,5}$. Subsequently, FDC was shown to possess broad substrate tolerance, decarboxylating differently (orto-, meta- or para-) substituted cinnamic acids, as well as its biaryl or heteroaryl derivatives ${ }^{2,6,7}$. Recently, FDC has been employed in an enzymatic cascade yielding stilbenes ${ }^{8}$ or shown to activate $\mathrm{C}-\mathrm{H}$ bonds through $\mathrm{CO}_{2}$ fixation, yielding unsaturated aromatic carboxylic acids ${ }^{9}$. Other studies investigated the effect of mutations/deletions of $f d c 1$ on the production of volatile phenols obtained during yeast alcoholic fermentation ${ }^{10}$, or quantitatively monitored the fermentation byproducts when $f d c 1$ in S. cerevisiae and S. eubazanus have been CRISPR edited ${ }^{11}$. The effect of $f d c 1$ single nucleotide polymorphisms (SNPs) on the decarboxylation activity of some industrially relevant yeasts was also reported ${ }^{12}$.

The stability issues of the prFMN cofactor ${ }^{13}$, as one the major factors limiting the applicability of FDC reactions, can be alleviated by the use of whole-cell ${ }^{6,7,14}$ or cell-free extract ${ }^{15} \mathrm{FDC}$-biocatalysts. Recently, several studies focused on the biosynthesis of the cofactor, aiming it's in vivo or in vitro synthesis ${ }^{16}$, that provides access to the fully active, holo-FDC. In Saccharomyces cerevisiae, the final step in the cofactor's biosynthesis, the prenylation

${ }^{1}$ Enzymology and Applied Biocatalysis Research Center, Faculty of Chemistry and Chemical Engineering, BabeșBolyai University, Arany János Street 11, 400028 Cluj-Napoca, Romania. ${ }^{2}$ These authors contributed equally: Horia Duță and Alina Filip. ${ }^{\bowtie}$ email: laszlo.bencze@ubbcluj.ro 
of FMN, is catalyzed by PAD1, a flavoprotein that is necessary for the generation of active, holo-FDC. In E. coli, UbiX substitutes PAD1 in its role of prFMN's biosynthesis, that serves as cofactor for UbiD, homologue of $\mathrm{FDC}^{17}$. As such, needing both $f d c 1$ and $p a d 1$ genes for the desired decarboxylase activity ${ }^{18}$, initially FDC has been studied in $S$. cerevisiae cultures, while later it has been shown that E. coli cultures expressing only the $f d c 1$ gene, due to the presence of UbiX of the host $E$. coli cells, can also function as whole-cell biocatalysts with the desired FDC-activity ${ }^{7,17-20}$. Supposedly, the cofactor's active iminium form is obtained inside FDC's active site under the influence of oxygen ${ }^{21}$ and a number of conserved residues ${ }^{22}$. However, in solution, $\mathrm{prFMNH}_{2} \mathrm{can}$ be irreversibly converted to inactive forms, such as prFMN C $\mathrm{ua}_{\mathrm{a}}-\mathrm{OOH}^{21}, \operatorname{prFMN} \mathrm{OH}, \operatorname{prFMN}_{\text {radical }}, \operatorname{prFMN}_{\text {radical }}-\mathrm{H}$, $\operatorname{prFMN}_{\text {iminium }}-\mathrm{OH}, \mathrm{C}_{1}$-ene-prFMN $\mathrm{iminium}^{1,13,23,24}$, while light decomposure has been also reported ${ }^{22}$, all these hindering the isolation of the holo-FDC.

Despite the cofactor stability issues, the identification of several novel decarboxylases harboring the prFMN cofactor $^{25-29}$, the broad substrate scope of FDC in comparison to phenolic acid decarboxylases from Enterobacter sp. or Bacillus pumilus $s^{30-32}$, or to benzoic acid decarboxylases ${ }^{33}$, that are limited to decarboxylations of $4-\mathrm{OH}$ cinnamates, or of benzoic acids, respectively, propelled FDC as one of the most versatile non-oxidative decarboxylases. Moreover, the high-quality crystal structures of FDC in apo-, holo- and ligand bound variants ${ }^{1,34-36}$ and the elucidation of the 1,3-cycloaddition reaction mechanism ${ }^{1-3}$, allowed initial rational design of FDC towards different substrates of interest, such as aromatic carboxylic acids, bulky cinnamic acid analogues and/ or aliphatic substrates $7,9,37,38$.

Encouraged by the versatility of FDC and its increasing biotechnological applicability, herein we provide a toolbox for the efficient laboratory evolution of FDC. Accordingly, we describe the development of a facile, fluorescent cell-plate FDC-activity assay, suitable for an initial, qualitative activity screen of mutant libraries generated through directed evolution or rational design based processes. In this work, the assay was validated by its employment within the activity screens of a focused FDC mutant library towards an aromatic substrate panel of high structural diversity. The increased activities of the FDC variants selected from the plate-assay, were confirmed by analytical scale biotransformations, while computational results supported and revealed the molecular level details of the improved enzyme activities. Notable, that despite the results represent an initial snapshot of employing the developed laboratory evolution toolbox for FDC, the correlation found between the nature/position of a certain functional group of the substrate and the corresponding active-site mutations, that improved the decarboxylation activity, also paves the way for the substrate-tailored protein engineering of FDC.

\section{Results and discussion}

Generation of substrate panel and focused FDC mutant library. Among the substrate panel (Fig. 1A) we included substrate analogues with ring-substituents of diverse electronic properties (1a-1e), or multiple substituted in various, $o-, m-, p$ - positions of the aromatic ring $(\mathbf{1 f}-\mathbf{1 i})$, as well as heteroaromatic $(\mathbf{1} \mathbf{j}$, $\mathbf{1 k}$ ), or differently connected bulky, biaryl (11-1o) or heteroaryl (1p) substrate analogues, of which active site orientations in several cases showed steric repulsions with active site residues of ScFDC. Notable, that compound 1p of the substrate panel was not transformed by wild-type FDC $^{7}$, while for bulky biaryl compounds (11-10) no proper active site orientations were obtained by the initial docking predictions. Besides the aim to assess the impact of substrate substitution pattern on the activity of the wild-type/mutant FDCs, upon substrate library generation the existing applications of the resulting styrenes were also considered. Accordingly, mono-substituted styrenes $\mathbf{2 a - 2 e}$ are building blocks in the synthesis of biologically active compounds ${ }^{39,40}$, while biaryl or heteroaryl styrenes $\mathbf{2 k}, \mathbf{2} \mathbf{l}$ are relevant for the synthesis of macromolecules ${ }^{41}$ or policyclic aromatic hydrocarbons ${ }^{42}$. Generally, the terminal double bond enables styrenes to act as versatile synthons in a variety of organic synthetic procedures, as reported in case of $\mathbf{2 d}-\mathbf{2} \mathbf{i}, \mathbf{2} \mathbf{o}^{43-45}$. Therefore, the employment/validation of the fluorescence-plate assay within the evolution of FDC towards these substrates intertwines with the synthetic value of the produced styrenes.

Further, we generated a focused ScFDC mutant library (Table S1), by the individual replacement of active site residues to smaller alanine or valine residues (Fig. 1B) to alleviate the steric repulsion of the targeted substrate panel, predicted by the docking studies using our computational model ${ }^{7}$. Residues involved in the reaction mechanism or cofactor/ substrate fixation, such as R175, E280 and E285 were not selected for mutations. Residue E285 is directly involved in the decarboxylation mechanism, having a role in the protonation step of the adduct obtained from the 1,3-dipolar cycloaddition between the C1' and C4a atoms of the prFMN cofactor and the $\alpha-\beta$ double bond of the substrate (Fig. 1C), step supposed to precede the Grob-type decarboxylative fragmentation that releases $\mathrm{CO}_{2}{ }^{1,2}$. Moreover, E285 and R175 interact with the carboxyl group of the substrate, while E280 has a direct influence on the pKa of both R175 and E285 (Fig. 1C). Thus, the active site residues selected for mutations include Q192 and I330, previously found to narrow the active site of ScFDC, hindering the accommodation of bulky non-linear substrates ${ }^{7}$. Since Q192 is also involved in cofactor binding through hydrogen bonding with the ribitol tail of prFMN (Fig. 1C and Fig. S33), besides mutation Q192A, variant Q192N was also considered. The remaining active site residues selected for mutations, such as I189, F397, I398, F440, M286 and L442 outline the hydrophobic region of the active site, accommodating the substrate's aryl moiety, and while not play an active part in the reaction mechanism, can impose steric constraints on the proper orientation ${ }^{1,7,22}$ of the differently substituted substrates, thus influencing reaction progress.

During the experimental work of our study, other studies implying protein engineering of $A n$ FDC and ScFDC has been reported, involving several active site residues of ScFDC such as F397, I398, M286 and I330, also selected by us for the mutant library generation. Accordingly, mutation of residues Y394, T395 of AnFDC and the corresponding homologous residues F397 and I398 of ScFDC provided FDC variants with decarboxylase activity for 1,3-cyclobutadiene ${ }^{37}$. Site-saturation mutagenesis at residues M283 and I327 of AnFDC (corresponding to M286 and I330 in ScFDC) provided FDC mutants with activity within the decarboxylation of atypic, benzoic acid-type 

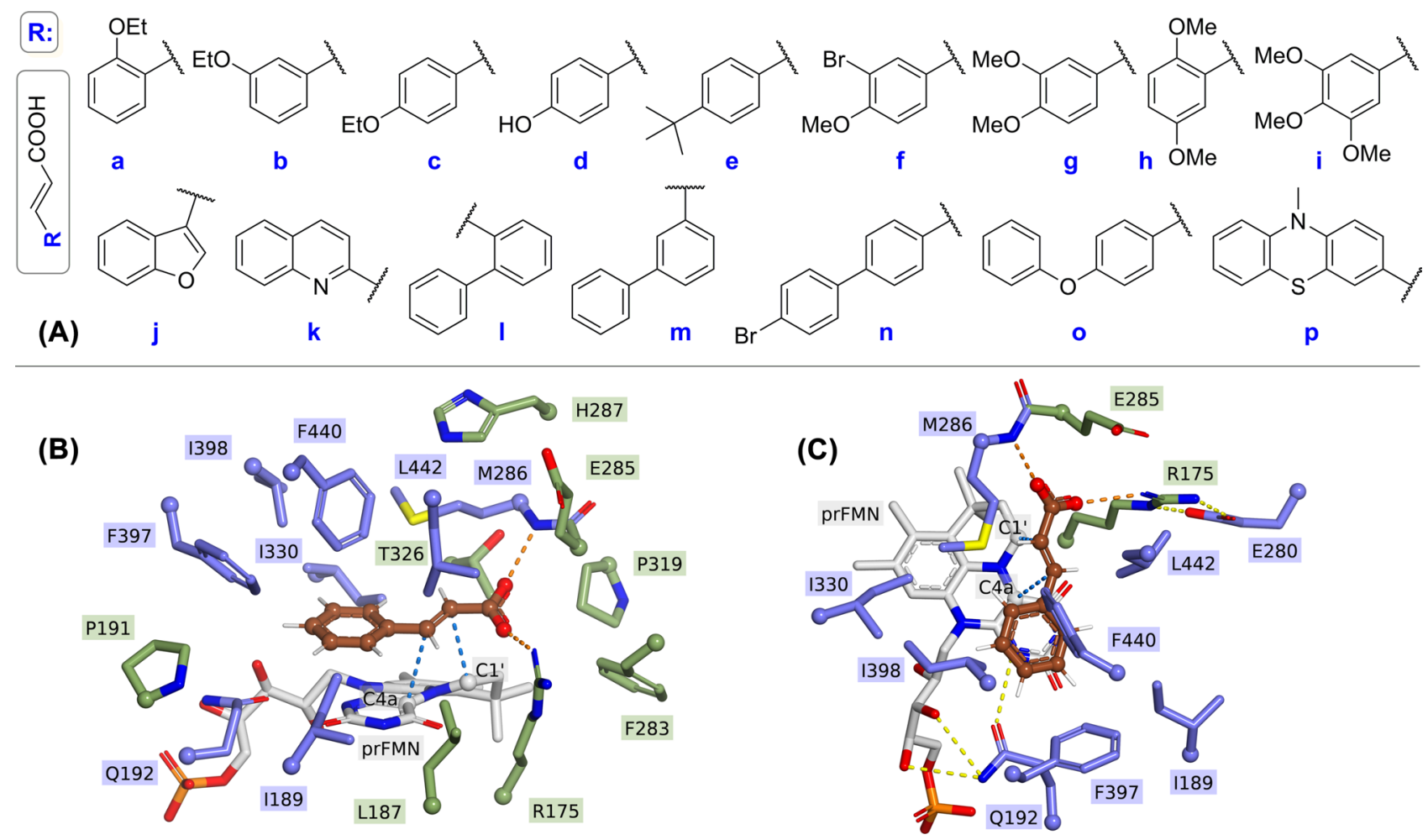

Figure 1. (A) substrate panel, including cinnamic acid derivatives 1a-p; (B) active site model of ScFDC (PDB: 4ZAC) accommodating trans-cinnamic acid as substrate, highlighting in blue the residues selected for individual replacement with alanine/valine $(\mathbf{C})$ top view of active site model with respect to the substrate plane: proper substrate binding requires the location of the $\alpha-\beta$ double bond of the substrate (highlighted in brown) in the proximity of carbon C1' and C4a atoms of the prFMN cofactor, that facilitates the 1,3-cycloaddition step of the reaction mechanism ${ }^{1,2}$. Hydrogen bonds between residues E280 and R175, as well as Q192 and the cofactor are shown as yellow dashed lines. Softwares used for the preparation of images are listed in Supporting information, Section 1.

aromatic substrates ${ }^{9}$. Besides these residues, our molecular docking predicted I189 as narrowing the catalytic site, providing steric repulsion in case of substrates with multiple substituted aromatic moieties, while replacement of bulky F440 residue in combination with L442 into smaller hydrophobic residues, was also considered. Notable, that all selected ScFDC active site residues, except F397 and I398 (corresponding to Y394 and T395 of AnFDC) are conserved within the two highly studied ScFDC and AnFDC variants (Table S2).

Cell-plate assay development. While the protein engineering of FDC is of high interest, for an efficient directed evolution-based engineering process, high-throughput activity assays, allowing facile activity screens of largely sized mutant libraries, are highly desirable. A plate assay, suitable for FDC activity screens at whole-cell level, also alleviates the tedious isolation process of holo-FDC ${ }^{1,19}$, however according to our knowledge, no such activity assay has been reported for FDC.

The 1,3-dipolar cycloaddition reaction between an alkene and a tetrazole represents an attractive method of fluorophore-forming bioorthogonal chemistry, with various diaryltetrazoles shown to be highly sensitive fluoroprobes for the detection of alkenes ${ }^{46-49}$. Furthermore, the photoinduced 1,3-dipolar cycloaddition using the selected diaryltetrazole (Fig. 2) as nitrile imine dipole was previously shown to possess compatibility for in vivo protein labeling within E. coli whole cells ${ }^{47-50}$. Building on this knowledge, recently, we developed a fluorescent phenylalanine ammonia-lyase (PAL) activity assay, that employs FDC as secondary, reporter enzyme, the produced styrene being fluorescently detected upon its reaction with a tetrazole-based fluorogenic probe $e^{51}$. Interestingly, at the same time a similar tetrazole-based assay was also reported for two operation modes of OleT decarboxylase, also validated on a focused mutagenesis library ${ }^{52}$. While both assays were optimized for use of cell-free extracts ${ }^{51}$, herein we focused on adapting the fluorescent detection of styrene derivatives to a cell-plate FDC-activity assay, providing facile, solid-phase decarboxylase activity screens (Fig. 2, steps 1-4).

Within the plate assay whole cells harboring the $f d c 1$ gene variants of the mutant library were used and tested in the decarboxylation reactions of the entire substrate panel. The plates were performed in duplicates using in each case as negative control, $E$. coli host cells without the plasmid containing $f d c 1$ gene. In the first step of the assay the cells of the different variants were grown, transferred onto a PVDF membrane, followed by IPTG induced-gene expression (Fig. 2). Cell permeabilization with chloroform, and a subsequent dialysis step of the membrane-attached colonies was followed by the decarboxylation reactions, performed by placing the membrane 
1. transfer of colonies to membrane

2. expression

3. cell lysis, dialysis

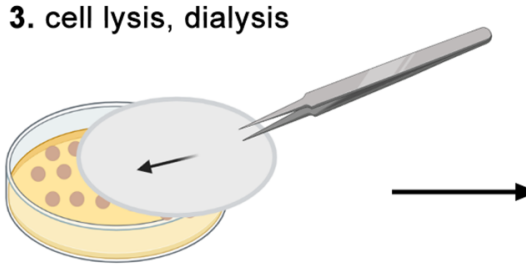

4. decarboxylation by FDC

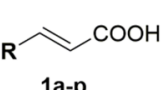

1a-p

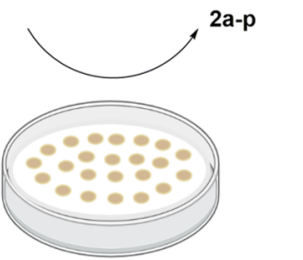<smiles>COc1ccc(-n2nnc(-c3ccccc3)n2)cc1</smiles>

$\mathrm{N}=\mathrm{N}$
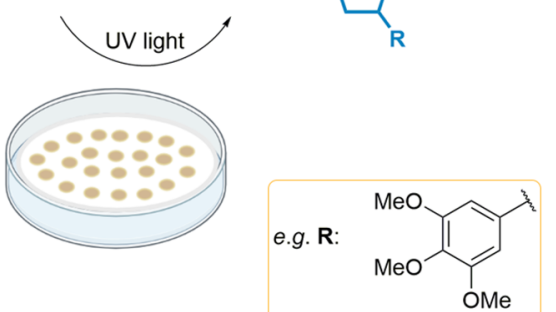

5. fluorogenic detection of styrenes

\begin{tabular}{|c|c|c|}
\hline FDC1 variant & RFI (\%)- Plate-assay & c (\%) - HPLC \\
\hline wt (wild-type) & 0 & $<1$ \\
\hline 23-F397Y/I189A & 100 & 83 \\
\hline 21-1398A/I189A & 83 & 78 \\
\hline 19-1330A/I398A & 50 & 65 \\
\hline 5-F397V/1398A & 28 & 60 \\
\hline $7-1330 \mathrm{~V} / 1398 \mathrm{~A}$ & 26 & 57 \\
\hline $1-1398 A$ & 26 & 57 \\
\hline $20-1398 \mathrm{~V} / \mathrm{I} 189 \mathrm{~A}$ & 18 & 54 \\
\hline $11-1330 \mathrm{~A}$ & 16 & 66 \\
\hline 13-I189A & 12 & \\
\hline 22-F397Y/I189V & 12 & \\
\hline 14-I189V & 8 & \\
\hline 15-F397Y & 4 & \\
\hline $18-F 397 \mathrm{~V} / 1398 \mathrm{~V}$ & 3 & \\
\hline
\end{tabular}

\section{monitoring by HPLC}

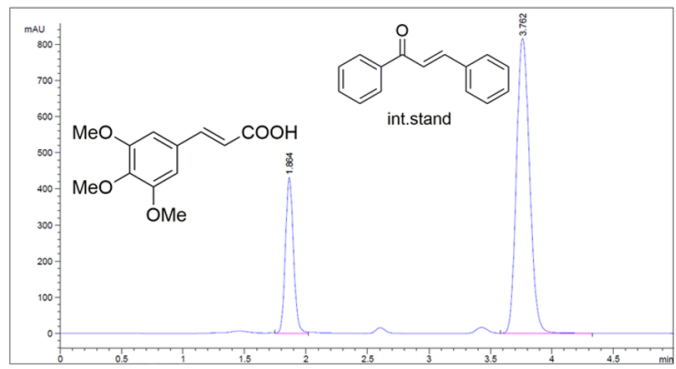

6. selection of hits

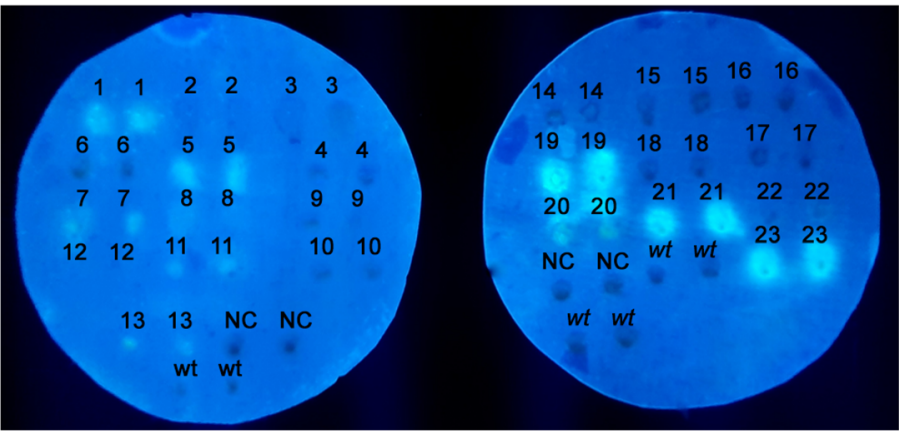

8. substrate depletion/conversion

\begin{tabular}{|l|r|}
\hline $6-L 442 V / F 440 A$ & 1 \\
\hline $2-F 397 A$ & 1
\end{tabular}

\begin{tabular}{|l|r|}
\hline $2-\mathrm{M} 286 \mathrm{~A}$ & 1 \\
\hline $12-1330 \mathrm{~V}$ & 1 \\
\hline
\end{tabular}

\begin{tabular}{|l|r|}
\hline $12-1330 \mathrm{~V}$ & 1 \\
\hline
\end{tabular}

$4-\mathrm{M} 286 \mathrm{~V}$

8-Q192A

9-Q192N

10-Q192S

$17-1398 \mathrm{~V}$

$16-F 397 \mathrm{~V}$

$\mathrm{RFI}$ - relative signal intensities; c - conversion values

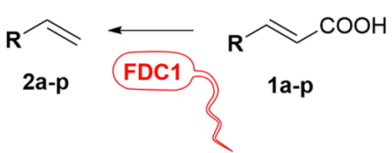<smiles>[R]C1CC(c2ccccc2)=NN1c1ccc(OC)cc1</smiles>

7. whole-cell FDC decarboxylations

Figure 2. Initial FDC activity screens using the fluorescent cell-plate assay allowing rapid identification of enzyme variants with improved activity, that upon selection have been characterized by the conversion values from their analytical scale decarboxylations. Representative results are presented in case of substrate analogue 3,4,5-trimethoxy-cinnamic acid $\mathbf{1 i}$ (for details of image preparations see Supplementary Information, Chapter 6) The cell-plate activity screens has been performed on the whole substrate panel 1a-1p (see Fig. S2-S17 and Tables S3-S5 for results of cell plate assays, while their detailed discussions in Sect. 2.3), while the conversions of the biotransformations of 1a-1p were determined by HPLC (Tables S7-S21) (Softwares used for image preparation are listed in Supporting information, Section 1).

onto the plate containing $2 \mathrm{mM}$ of the corresponding substrates $\mathbf{1 a}-\mathbf{1 p}$, followed by incubation at $37^{\circ} \mathrm{C}$ for $4 \mathrm{~h}$. For the subsequent fluorogenic reaction, incubation of the membrane with the tetrazole fluoroprobe and UVirradiation was employed, followed by the detection of the fluorescence signal through an imaging system (Fig. 2).

FDC-activity screens and HPLC conversions. The developed cell-plate assay has been employed for the initial, qualitative assessment of the enzyme activities of the FDC mutant library towards the substrate panel, allowing the selection/identification of the best performing variants, based on their relative fluorescence signal intensities (Tables S3-S5, Fig. S2-S17). Further, the selected best performing variants and several non-reporter variants were used as induced whole cell biocatalysts in analytical scale biotransformations of the corresponding substrates, monitoring the conversion values by reverse phase HPLC to provide their conversion-based activity order (Tables S7-S21, Fig. 3). In case of substrates, 1a-1f, 1h, 1i, 11, 1n the activity-order of the variants based on the fluorescent signal intensities from the cell-plate assay were in good correlation with the conversion-based activity ranking of the hits selected from mutant library (Fig. 3 and Tables S7-S12, S14, S15, S18, respectively). Accordingly, the best three-four responders from the plate assay provided the highest conversions of substrates 1a-1f, $\mathbf{1 h}, \mathbf{1 i}, \mathbf{1 1}, \mathbf{1 n}$, while the variants exhibiting no fluorescence signal were also inactive or resulted in low conversions $(<8 \%)$ within the HPLC-monitored biotransformations (Tables S7-S12, S14, S15, S18). Among these results, notable, the presence of some exceptions such as the case of variant F397Y/I189A, providing high 


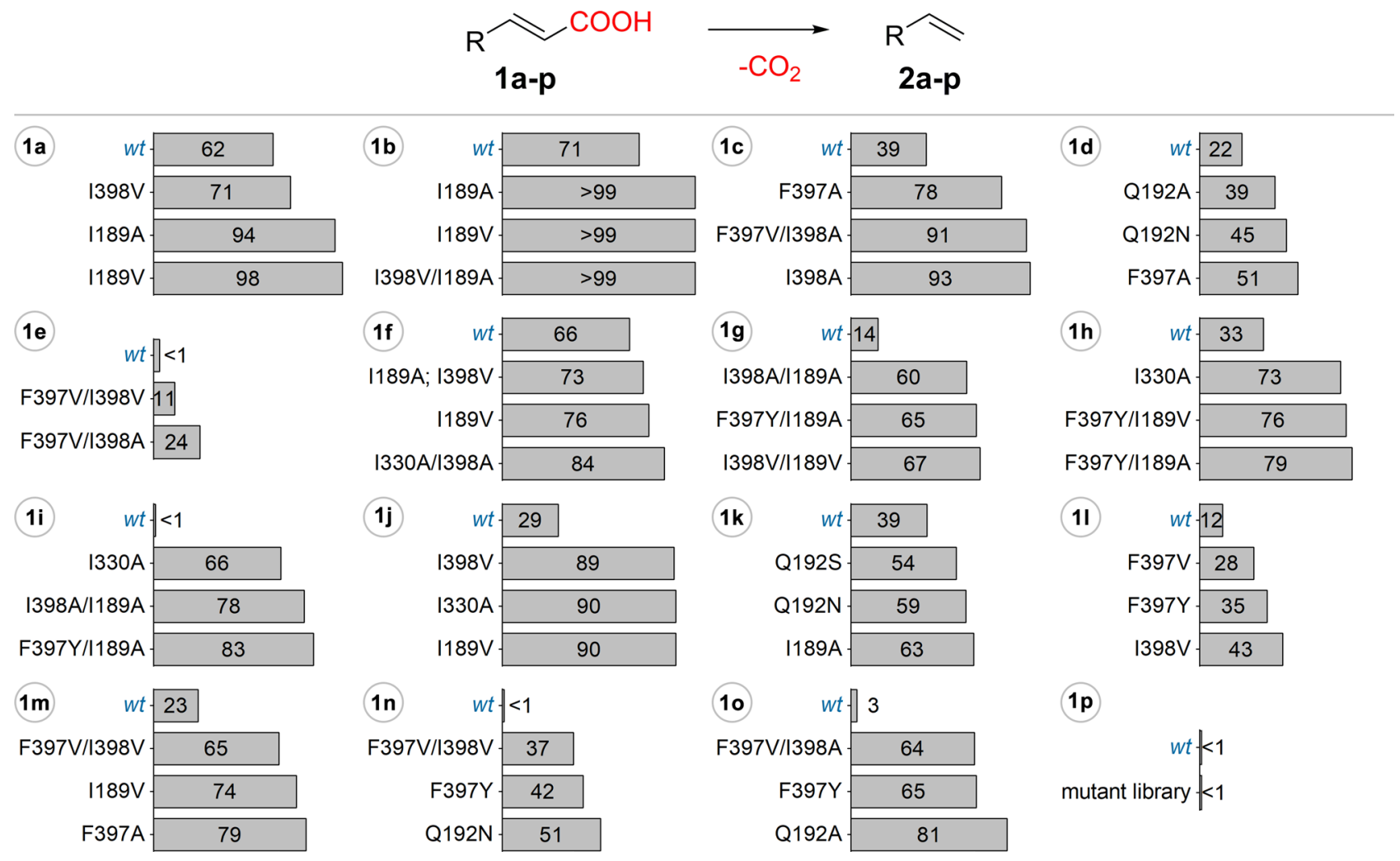

Figure 3. HPLC conversion values obtained from the analytical scale biotransformation of the substrate panel 1a-1p, using ScFDC whole-cell biocatalyst. Results of the best performing three variants and of the wild-type ScFDC are shown. Softwares used for the preparation of images are listed in Supporting information, Section 1.

fluorescent signal intensity (95\% RFI) in the plate-assay and moderate conversions (44\%) in case of substrate $\mathbf{1 f}$ (Table S12), or variant I330A with low fluorescent intensity (16\% RFI) and high conversion values of $66 \%$ in case of $\mathbf{1 i}$ (Table S15). Besides, moderate correlation has been observed in case of substrates 1k (Table S17), the good reporter variants (RFI $>50 \%$ ) providing the highest conversions values of $>52 \%$ ), but moderate reporter variants M3 and M1 (18\% and 21\% RFI) showed lower conversion values (<6\%) than the weak reporter variant M17 ( $12 \%$ conversion and RFI of $5 \%$ ). While in case of substrates $\mathbf{1 g}, \mathbf{1 j}, \mathbf{1 m}$, and $\mathbf{1 0}$ no clear correlation of the activity-order could be observed, the cell-plate assay still provided a suitable "yes/no"-type response for initial activity screens using substrates $\mathbf{1 g}, \mathbf{1 j}$ and $\mathbf{1 0}, 24$ out of 27 colonies, acting as good reporters (RFI $>50 \%)$ in the plate assay, showed significantly increased conversions in the analytical scale biotransformations over those obtained by the low/non-reporter colonies $(\mathrm{RFI}<10 \%)$, (Tables S13, S16, S21). In case of $\mathbf{1 m}$, variants with improved conversions (M2, M13, M14, M18) could also be identified among the good reporters (RFI > 50\%), but M16 acting as non-reporter within the plate assay provided conversion of $17 \%$, while moderate/good reporter variants M9, M19 and M5 (RFI\% of 26, 59 and 37, respectively) showed low HPLC conversions of 4-9\% (Table S19). The occurrence of similar false positive hits is also observed at the structurally similar, biphenyl derivative 1n, in which case variants $w t$, M19 and M23 of low to moderate fluorescence intensities of $11-21 \%$ RFI, showed no conversions within the biotransformations (Table S20). In these cases, the increased background fluorescence and low substrate solubilities, combined with cell-growth differences of the colonies from the assay-plate might induce the appearance of false positive hits. Finally, in the particular case of $\mathbf{1 p}$, the high background fluorescence of the phenothiazine moiety, hindered the cell-plate activity screens (Fig. S17), thus the analytical scale biotransformations of $\mathbf{1 p}$ have been performed with the whole mutant library, however none of the ScFDC variants provided detectable conversions.

These results obtained on a diverse set of cinnamic acids support that the plate-assay is suitable for initial, qualitative-type FDC-activity assessments at whole-cell level, required within the high-throughput screens of the directed evolution-based enzyme engineering. While for several substrates $\mathbf{1 a}-\mathbf{1 f}, \mathbf{1 h}, \mathbf{1 i}, \mathbf{1 l}, \mathbf{1 n}$ the activity rank of the mutant library obtained with the plate assay resembled the conversion-based activity order, the low-degree correlation obtained in case of substrates $1 \mathbf{g}, \mathbf{1 j}, \mathbf{1 m}, \mathbf{1 0}$, recommends the use of the plate assay for activity screens with a "yes/no"-type response, which allows the detection of hits with decarboxylase-activity. This can be followed by activity order/catalytic efficiency assessments of the hits of decarboxylase activity using the available HPLC methods, which on the other hand, are not suitable for the high-throughput activity screens of large mutagenesis libraries. Moreover, the dysfunctionality of the plate assay or appearance of false positive hits for substrates with significant structural differences to cinnamic acid, e.g. 1p, 1 m reveals the limitations of the 
current form of the plate assay, that employs the optimal detection conditions developed for the decarboxylation of cinnamic acid ${ }^{51}$, and highlights 'substrate-personalization' perspectives.

Furthermore, is highly notable, that the activity screens/conversion assessments revealed several "hot-spot" active site residues, which upon mutations resulted in FDC variants of improved activity. Interestingly mutation of residue F397 of ScFDC to tyrosine, its homologue residue from AnFDC, in several cases, such as decarboxylations of 1c, 1g, 11, 1n and 1o, provided increased fluorescent signals (Figures S4, S8, S13, S15, S16, respectively and/or Tables S3, S4, S5) and conversions (Tables S9, S13, S18, S20, S21 respectively and/or Fig. 3). Mono-ethoxy substituted cinnamic acids $\mathbf{1 a}$ and $\mathbf{1 b}$, transformed by wild-type ScFDC with moderate/good conversions of $62 \%$ and $71 \%$ (Fig. 3), respectively, were quantitatively decarboxylated by variants I189A/V, suggesting a preferable orientation of the orto-, meta- substituents towards residue I189. In case of para-substituted substrates 1c-1e, mutation of residues F397 and I398 improved the enzyme activity, resulting in conversions up to $78 \%$ and $93 \%$, respectively, in comparison with the $39 \%$ (for 1c) and $22 \%$ (for 1d) conversions provided by the wild-type enzyme (Fig. 3). The sterically more demanding tert-butyl substituted derivate 1e was not transformed either by wild-type or single mutants F397V or I398A(V) variants, however double mutant variants F397V/I398A and F397V/I398V provided conversions of $11 \%$ and $24 \%$, respectively, revealing an additive effect of the combined mutations. The active site orientation of the substrate's aromatic substituents, depicted by the biotransformations of mono-substituted derivatives, were also supported by the decarboxylations of the disubstituted substrates $\mathbf{1 f}-\mathbf{1 h}$. Notable, that substrate analogues $\mathbf{1 f}$ and $\mathbf{1 g}$, disubstituted in the meta, para-position of the aromatic ring, resemble the substitution pattern of the natural substrate, ferulic acid. Thus, expectedly wild-type ScFDC transforms all three disubstituted substrates, obtaining high conversions of $66 \%$ in case of $\mathbf{1 f}$, and moderate/ low conversions for the dimethoxy-cinnamic acids (14\% in case of $\mathbf{1 g}$, and $33 \%$ in case of $\mathbf{1 h}$ ). In accordance with previous reports ${ }^{2,7}$, this result supports that electronic effects also influence the enzyme activity of FDC. However, aiming to find residues, of which mutations lead to improved activities, we continued to focus on the relative activity increment within the variant library towards each individual substrate. Accordingly, the significant improvements in conversion values in case of 3,4-dimethoxy-cinnamic acid $\mathbf{1 g}$ when using mutant variants I189A/V and I398A (increase with 45-52\% in comparison with wild-type-Fig. 3, Table S13), corresponds with the supposed orientation of the meta- and para-substituents towards residues I189 and I398, respectively. In case of 2,5-dimethoxy-cinnamic acid $\mathbf{1 h}$, besides the expected superior activity of I189A and I398A/I189A variants, reflected in an increase of conversion values with $29 \%$ and $33 \%$, relative to those obtained with the wild-type FDC, variant I330A provided the highest activity increase $\left(c_{1330}=73 \%\right.$, while $\left.c_{w t}=33 \%\right)$ among the single mutants (Table S14). This suggests, that in case when substituents occupy both ortho-, or meta-positions from the different sides of the aromatic ring, two active site residues, namely I189 at one side and I398/I330 on the other side of the active site, are involved in the substituent's accommodation. Notable, that also in case of $\mathbf{1 h}$, mutation F397Y, similarly to the decarboxylation of $\mathbf{1 c}, \mathbf{1 g}, \mathbf{1 1}, \mathbf{1 n}$ and 1o, provided activity increase, in this case additional, for the single mutant I189A, leading to variants F397Y/I189A(V) with improved conversions of 79 and $76 \%$ (Fig. 3, Table S14). The biotransformations of the 3,4,5-trimethoxy-cinnamic acid 1i further supports these correlations, while wild-type FDC proved to be inactive, double mutant variants, such as I330A(V)/I398A, I398A(V)/I189A or F397V(Y)/I398A, including mutations of residues I398, F397 (for para-positioned substituents) or I189, I330 (for meta-substituents) provided superior variants, resulting in conversions of 54-83\% (Figs. 2, 3, Table S15).

Definitely, substrate accommodation of bi(hetero)-aromatic substrate analogues $\mathbf{1 j}-\mathbf{1} \mathbf{p}$ is less predictable based on the active site model (Fig. 1), therefore the mutation-activity increase correlation, revealed by the activity measurements, is even more valuable for the structure-guided protein engineering of FDC. Despite that heteroaromatic, bicyclic derivatives $\mathbf{1} \mathbf{j}$ and $\mathbf{1 k}$ show a different structural architecture, with functionalization occurring in the 3' and 2' positions of the heteroaryl rings, similar mutations of residues I189, I330 provide variants I189A(V) and I330A, with superior conversions of $\sim 90 \%(\mathbf{1 j}), 45-63 \%(\mathbf{1 k})$ to the moderate conversions of $29 \%(\mathbf{1 j})$ and 39\% (1k) obtained by the wild-type FDC (Table S16, S17, respectively and Fig. 3). Regarding the transformations of bulkier, differently connected biarylic substrate analogues 11-1o, wild-type ScFDC showed low ( $12 \%$ and $23 \%$ conversions for $\mathbf{1 1}$ and $\mathbf{1 m}$, respectively-Tables S18, S19) or no activity (1), 1o-Tables S20, S21). Besides the expected beneficial effect of individual or combined mutations of F397A and I398A(V), providing superior variants with good to moderate conversions, mutant variant F397Y also resulted in improved $41.5 \%$ and $65.3 \%$ conversions for $\mathbf{1 n}$ and 1o, respectively. Generally, in case of these bulky substrates 11-10, mutations of similar hot-spot residues (I189, Q192, F397, I398) provided single/double mutant variants of high activity, leading to conversions between 37 and $81 \%$ for compounds $\mathbf{1 m - o}$ (Fig. 3, Tables S19-S21).

Accordingly, the activity screens of the FDC variant library towards the substrate panel provided a comprehensive active site map, which correlates the substitution pattern of the substrate's aromatic moiety with its specific active site positioning, strengthening the rational design-based FDC engineering.

Computational studies. Based on previous reports ${ }^{1,7,9}$ and ligand bound AnFDC crystal structures (PDB ID: $4 \mathrm{ZA7}, 4 \mathrm{ZA} 8,4 \mathrm{ZAB})$, the proper binding of the substrates implies several requirements. Among them, the location of the $\alpha-\beta$ double bond of the substrate should be in the proximity of the C1' and C4a atoms of the cofactor, necessary for the 1,3-dipolar cycloaddition mechanism (Fig. 1 and Fig. S23). Furthermore, in ScFDC, R175 and E285 interact with the carboxyl group of the substrate, while E285 acts also as acid-base in the reaction mechanism, while E280, tunes the pKa of R175 and in turn E285 (Fig. S23) ${ }^{22}$. Accordingly, the reaction rates are influenced by multiple substrate-related factors, such as inductive effects of substituents, presence of extended conjugation, substrate orientation related to the prFMN and within the catalytic site, that is influenced by both the size and planarity of the substrate. Considering these factors and using our previously validated molecular docking method $^{7}$, we attempted to gain molecular level insights on the beneficial effect of the mutations on enzyme activities. 

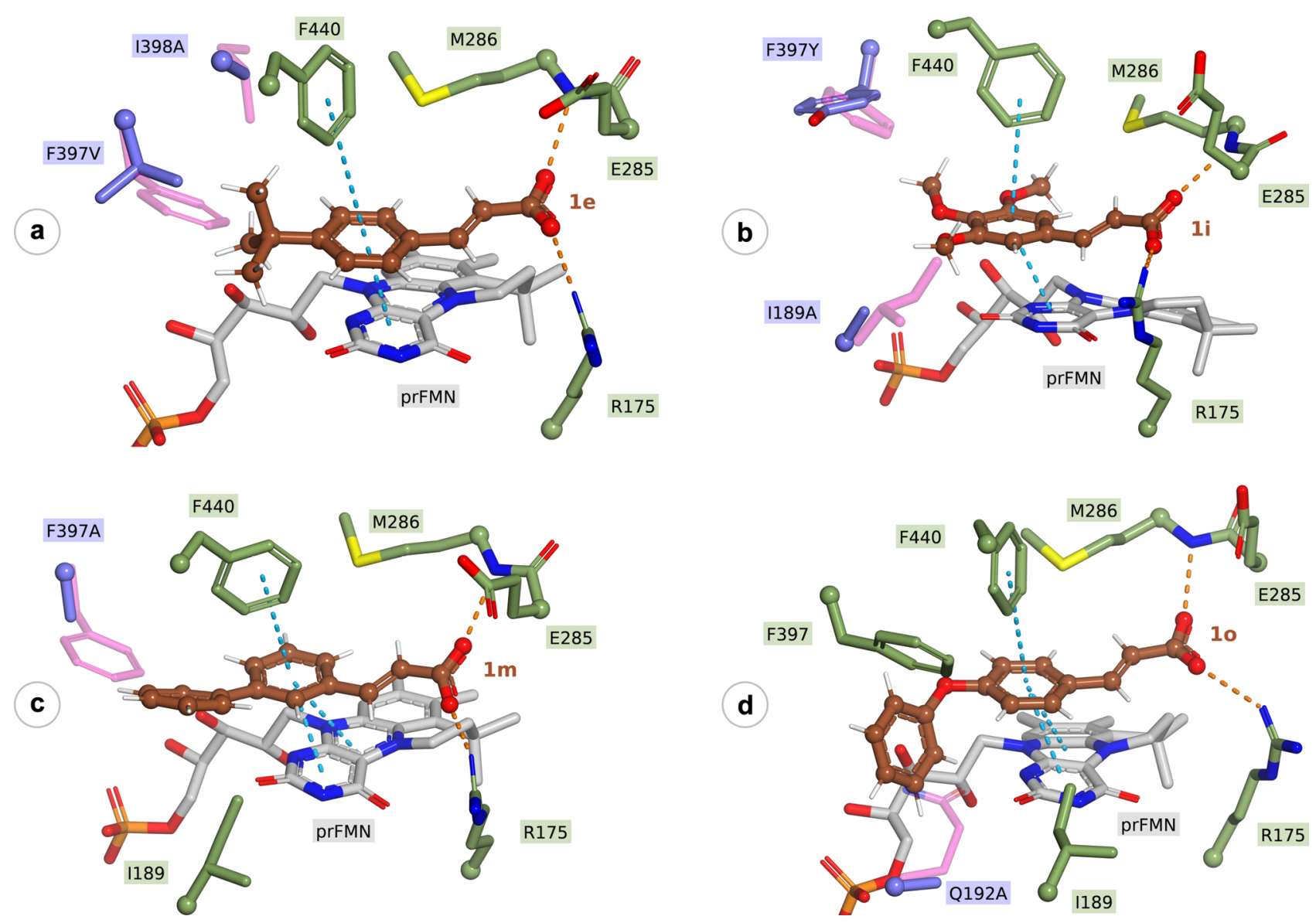

Figure 4. In each figure the side chains of the preserved active site residues of ScFDC1 (green) and the mutant residues (blue) overlaid with their original counterparts from the wild-type enzyme (magenta) are represented as stick models. The substrates $\mathbf{1 e}, \mathbf{1 i}, \mathbf{1 m}, \mathbf{1 0}$ are colored in brown within Fig. 4a-d, respectively. Hydrogen bonds between the carboxyl group of the substrate and the backbone nitrogen atom of residue M286 and side chain of residue R175 are indicated as orange dashed lines, whereas the blue dash corresponds to the pi-pi interactions of the substrate's aromatic ring and residue F440 and the prFMN cofactor, respectively. All these interactions were considered within the selection process of the proper binding state, required for the 1,3-cycloaddition mechanism. Softwares used for the preparation of images are listed in Supporting information, Section 1.

Generally, the obtained computational results were in good agreement with the experimentally observed activity enhancements, revealing proper substrate orientations within the catalytic site of the best performing mutant variants. In the following, representative cases are presented, supporting that in contrast to the wildtype ScFDC, appropriate mutations relieved the steric hindrance between the substrate and the side chain of the corresponding active site residues. Substrate positioning of representative compounds $\mathbf{1 e}, \mathbf{1 i}, \mathbf{1} \mathbf{m}, \mathbf{1 0}$ within the active site of the corresponding best performing FDC variants is in good agreement with the optimized model of the cinnamic acid bound into the wild-type ScFDC1 (Fig. S24).

In the case of $p$-(tert-butyl)cinnamic acid 1e, converted only by variants F397V/I398A and F397V/I398V (Fig. 3), proper binding orientations of 1 e have been obtained within the catalytic site of both variants (Fig. 4a), while within the wild-type ScFDC no active substrate-binding state could be observed. The inactivity of the $w t$ ScFDC can be attributed to the steric clash between the tert-butyl group of substrate $1 \mathbf{e}$ and the aromatic ring of F397, shown in magenta in (Fig. 4a).

In the case of 3,4,5-trimethoxy-cinnamic acid 1i, the side chains of residues I330 and I189 impede the approach of the substrate to the binding site. The atomic overlap between one of the meta-positioned methoxy group of the substrate and the side chain of I189 (shown in magenta) can be observed in Fig. $4 \mathrm{~b}$, where the optimized model of the best performing F397Y/I189A variant is represented.

For bulky substrate $\mathbf{1 m}$, within the best performing variants, mutation F397A provides space for the biaryl ring system, allowing the proper orientation of the double bond for the 1,3-cycloadditions (Fig. 4c). The mutation relieved the steric hindrance between the two aromatic rings of the F397 side chain and substrate 1m, which can be noticed in Fig. 4c. It is noteworthy that this conformation of the substrate corresponds to the ground state geometry, which could explain the improved conversions observed using variant F397A $(c=79 \%)$ with respect to the wild-type enzyme $(\mathrm{c}=23 \%)$. 
In case of the similarly bulky substrate 1o, the etheric group bends the two aromatic rings relatively to each other, resulting in a partial overlap between the $p$-phenoxy substituent group and residue Q192, explaining the enhanced activity of variant Q192A (Fig. 4d). Unfortunately, computational results didn't provide molecular level evidence for the improved activity of variant F397Y.

The strong correlation of the experimental and computational data suggests that the employed molecular model is suitable also for in-silico activity predictions for ScFDC, providing a computational tool for the rational engineering of FDC. Notable, that during the preparation of our manuscript in silico activity predictions were also reported in tailoring AnFDC and ScFDC for the 1,3-butadiene synthesis ${ }^{37}$.

\section{Conclusions}

Within our study a fluorescent plate assay suitable for the high-throughput (HT) activity screens for ferulic acid decarboxylases (FDCs), has been developed and validated through the activity screens of a mutant library rationally designed towards a 16-membered aromatic substrate panel. Identification of mutant variants of enhanced activities provided correlation between the substitution pattern of substrates with their specific active site accommodation. The computational data supported the experimentally observed activity enhancements and revealed proper substrate orientations within the catalytic site of the best performing mutant variants and supported our molecular model for in silico FDC-activity predictions. Accordingly, the computational activity predictions, the developed HT-activity assay and the identified substrate specificity modulator residues provide a powerful toolbox for the directed evolution or rational designed based protein engineering of FDCs.

\section{Experimental part \\ Materials and methods. See detailed description in Supporting Information, Chapter 1 and Chapter 2.}

Mutant library generation. The FDC variant library was obtained through site-directed mutagenesis as described in Supporting information, Chapter 5.

Substrate panel generation. The tested substrate library was obtained through the Knoevenagel-Doebner reaction using the corresponding aromatic aldehydes as starting material. For the detailed description of the procedures, obtained yields and NMR data see Supporting information, Chapter 3.

Initial activity screens with cell-plate assay. For all assay plate, whole-cells of $E$. coli Rosetta (DE3) pLysS were used as expression hosts, harboring the pCDF-Duet1 plasmid carrying the genes of Scfdc1 and Scpad1 (for detailed description of the plasmid construction, molecular cloning see ESI, Chapter 4).

$2 \mu \mathrm{L}$ of cell suspensions harboring the plasmids of each FDC variant were transferred (pipetted) onto LB-agar Petri plates containing chloramphenicol $(34.0 \mu \mathrm{g} / \mathrm{mL})$, followed by overnight incubation at $37^{\circ} \mathrm{C}$. The colonies grown on the agar plate were transferred on a PVDF membrane, pre-treated by washing with methanol and phosphate buffer (100 $\mathrm{mM} \mathrm{NaH}_{2} \mathrm{PO}_{4} \mathrm{pH}$ 7.0). For successful colony transfer the membrane was left for $20 \mathrm{~min}$ on the plate. Further, the membrane was transferred to an induction plate (LB-agar with $1 \mathrm{mM}$ IPTG and $34.0 \mu \mathrm{g} / \mathrm{mL}$ chloramphenicol) and incubated for $8 \mathrm{~h}$ at $30^{\circ} \mathrm{C}$. Cell permeabilization was performed by placing the membrane under chloroform vapours for $45 \mathrm{~s}$ using a desiccator, followed by dialysis on $0.4 \%$ agarose plate in phosphate buffer $\left(100 \mathrm{mM} \mathrm{NaH}_{2} \mathrm{PO}_{4} \mathrm{pH} 7.0\right)$ and storage at $4{ }^{\circ} \mathrm{C}$, overnight.

The reaction medium plate was prepared by dissolving substrates $\mathbf{1 a}-\mathbf{p}$ at $1 \mathrm{mM}$ final concentration in $1 \%$ agarose gel, followed by the incubation of the membrane on the reaction plate at $37^{\circ} \mathrm{C}$ for $4 \mathrm{~h}$. For the fluorescent detection of the colonies with FDC activity, the membrane was placed on a filter paper moistened with a solution of $100 \mathrm{M}$ tetrazole in phosphate buffer $\left(100 \mathrm{mM} \mathrm{NaH}_{2} \mathrm{PO}_{4}, \mathrm{pH} 7.0\right)$ and incubated in dark for $1 \mathrm{~h}$ at $37^{\circ} \mathrm{C}$, followed by UV-irradiation at $302 \mathrm{~nm}$ for $1 \mathrm{~min}$. The detection of signal intensities provided by the colonies of the assay plate was performed by ChemiDoc ${ }^{\mathrm{Tm}}$ Imaging System, using a corresponding UV filter, allowing detection of specific emissions at $>360 \mathrm{~nm}$ wavelengths. The obtained images were analyzed by the Image Lab 5.2.1 software, selecting an area of $2.8 \mathrm{~mm}^{2}$ from each spot corresponding to the different colonies, for which the background given by the negative control colony has been decreased from the mean values of all pixels inside the boundary volume. The obtained maximum signal intensity value being considered as $100 \%$ relative enzyme activity of the other signal intensities provided the corresponding relative activities. All assay-plates have been performed in duplicates, and in all plates the negative controls were represented by the E. coli host cells, harbouring the empty pCDF-DUET1 vector.

Analytical scale biotransformations. Culture preparation. Cultures of E. coli BL21(DE3) cells were prepared using Luria-Bertani (LB) medium supplemented with chloramphenicol and streptomycin, that was inoculated with $1-2 \mathrm{v} / \mathrm{v} \%$ of overnight culture. Following incubation at $37^{\circ} \mathrm{C}, 220 \mathrm{rpm}$, the cultures were induced with IPTG (at a final concentration of $0.2 \mathrm{mM}$ ) at $\mathrm{OD}_{600} \sim 0.6$, followed by incubation at $25^{\circ} \mathrm{C}, 220 \mathrm{rpm}$ until to a cell density of $\mathrm{OD}_{600} \sim 2$. The cells were harvested via centrifugation and immediately used in biotransformations.

Analytical scale FDC mediated decarboxylations. Stock solutions of each substrate in DMSO (50 mM) were diluted to $2 \mathrm{mM}$ or to $1 \mathrm{mM}$ in the case of substrates of low solubility, $\mathbf{2 k}-\mathbf{p}$, with phosphate buffer $(100 \mathrm{mM}$ $\mathrm{NaH}_{2} \mathrm{PO}_{4}, \mathrm{pH} 7.0$ ). The freshly prepared, induced E. coli cells harboring $f d c 1$ genes, were resuspended in the reaction solution to a final $\mathrm{OD}_{600}$ of 2 , followed by incubation of the reaction mixtures at $35^{\circ} \mathrm{C}, 700 \mathrm{rpm}$, for $16 \mathrm{~h}$. 
RP-HPLC monitoring. After $16 \mathrm{~h}$ of reaction time, the entire reaction mass was subjected to cellular lysis through sonication, followed by the removal of the cellular pellet through centrifugation at 13,400 rpm, 12,000 g, for $10 \mathrm{~min}$. The cellular pellet was extracted with $500 \mu \mathrm{L} \mathrm{MeOH}$, which was combined with the supernatant of the previous centrifugation step. $100 \mu \mathrm{L}$ of the combined solution was diluted with $100 \mu \mathrm{L}$ of a solution containing $100 \mathrm{mM} \mathrm{NaH}_{2} \mathrm{PO}_{4}, \mathrm{pH} 7.0$, acetonitrile, and benzalacetophenone, used as an internal standard (please refer to Supporting information, Chapter 7., HPLC methods for the exact composition of this solution). All HPLC analyses were performed at $25^{\circ} \mathrm{C}$ using a Phenomenex Kinetex NX-C18 $150 \times 4.5 \mathrm{~mm}$ column, a mobile phase of $30 \% \mathrm{H}_{2} \mathrm{O}(0.1 \% \mathrm{v} / \mathrm{v}$ TFA $)$ and $70 \% \mathrm{ACN}(0.1 \% \mathrm{v} / \mathrm{v} \mathrm{TFA})$, a flow-rate of $1 \mathrm{~mL} / \mathrm{min}$, injecting $5 \mu \mathrm{L}$ of the previously obtained samples. Conversion values were determined by monitoring the depletion of the substrate concentration, using benzalacetophenone as an internal standard (for detailed description of conversion determination and relative response factors see Supporting Information, Chapter 7).

Molecular docking. The molecular docking calculations were carried out by the Autodock Vina software ${ }^{53}$, using flexible-ligand and rigid-receptor docking. The docking parameters were modified to ensure that among the resulting poses the one with proper binding orientation of the substrate (as defined previously in Section 2.4) could be identified. Accordingly, a longer search was employed by setting the exhaustiveness of the search to 100, whereas the energy range between the best and worst binding pose was adjusted to $10 \mathrm{kcal} / \mathrm{mol}$. The dimension of the search space was defined by the binding site residues highlighted in Fig. 1 and the prFMN cofactor, based on the crystal structure of $S c F D C 1$ (PDB: $4 Z A C)^{1}$, therefore a cubic grid box with the size of $18 \AA \times 18 \AA \times 18 \AA$ was employed as it can be seen in Figure S25.

The ground state geometries of the substrates were obtained by means of density functional theory. All quantum chemical calculations were performed by Gaussian $09^{54}$ employing the B3LYP density functional with the $6-31 \mathrm{G}(\mathrm{d}, \mathrm{p})$ basis set, in a water solvated environment using the Polarizable Continuum Model $(\mathrm{PCM})^{55}$.

The crystallographic structure of $S c F D C 1$ was retrieved from Protein Data Bank entry 4ZAC ${ }^{1}$. The inactive conformation of residue E285 was altered according to those observed in the ligand bound AnFDC1 crystal structures. The selected docking results were submitted for minimization using the YASARA web server ${ }^{56}$.

Received: 14 September 2021; Accepted: 19 January 2022

Published online: 01 March 2022

\section{References}

1. Payne, K. A. P. et al. New cofactor supports $\alpha$, $\beta$-unsaturated acid decarboxylation via 1,3-dipolar cycloaddition. Nature 522, 497-501. https://doi.org/10.1038/nature14560 (2015).

2. Ferguson, K. L., Arunrattanamook, N. \& Marsh, E. N. G. Mechanism of the novel prenylated flavin-containing enzyme ferulic acid decarboxylase probed by isotope effects and linear free-energy relationships. Biochemistry 55, 2857-2863. https://doi.org/10. 1021/acs.biochem.6b00170 (2016).

3. Ferguson, K. L., Eschweiler, J. D., Ruotolo, B. T. \& Marsh, E. N. G. Evidence for a 1,3-dipolar cyclo-addition mechanism in the decarboxylation of phenylacrylic acids catalyzed by ferulic acid decarboxylase. J. Am. Chem. Soc. 139, 10972-10975. https://doi. org/10.1021/jacs.7b05060 (2017).

4. McKenna, R. \& Nielsen, D. R. Styrene biosynthesis from glucose by engineered E. coli. Metab. Eng. 13, 544-554. https://doi.org/ 10.1016/j.ymben.2011.06.005 (2011).

5. McKenna, R., Thompson, B., Pugh, S. \& Nielsen, D. R. Rational and combinatorial approaches to engineering styrene production by Saccharomyces cerevisiae. Microbial Cell Factories 13, 1-12. https://doi.org/10.1186/s12934-014-0123-2 (2014).

6. Aleku, G. A. et al. Terminal alkenes from acrylic acid derivatives via non-oxidative enzymatic decarboxylation by ferulic acid decarboxylases. Chem CatChem 10,3736-3745. https://doi.org/10.1002/cctc.201800643 (2018).

7. Nagy, E. Z. A. et al. Exploring the substrate scope of ferulic acid decarboxylase (FDC1) from Saccharomyces cerevisiae. Sci. Rep. 9, 1-10. https://doi.org/10.1038/s41598-018-36977-x (2019).

8. Mertens, M. A. S. et al. Chemoenzymatic cascade for stilbene production from cinnamic acid catalyzed by ferulic acid decarboxylase and an artificial metathease. Catal. Sci. Technol. 9, 5572-5576. https://doi.org/10.1039/c9cy01412h (2019).

9. Aleku, G. A. et al. Enzymatic C-H activation of aromatic compounds through $\mathrm{CO}_{2}$ fixation. Nat. Chem. Biol. 16, 1255-1260. https://doi.org/10.1038/s41589-020-0603-0 (2020).

10. Ogata, T., Yamada, R., Ayuzawa, R. \& Nakamura, K. Mutation and deletion of PAD1 and/or FDC1 and absence of phenolic offflavor production in top- and bottom-fermenting yeasts. J. Am. Soc. Brew. Chem. 78, 74-79. https://doi.org/10.1080/03610470. 2019.1678911 (2020).

11. Mertens, S. et al. Reducing phenolic off-flavors through CRISPR-based gene editing of the FDC1 gene in Saccharomyces cerevisiae $\mathrm{x}$ Saccharomyces eubayanus hybrid lager beer yeasts. PLOS ONE 14, 0209124. https://doi.org/10.1371/journal.pone.0209124 (2019).

12. Mukai, N., Masaki, K., Fujii, T. \& Iefuji, H. Single nucleotide polymorphisms of PAD1 and FDC1 show a positive relationship with ferulic acid decarboxylation ability among industrial yeasts used in alcoholic beverage production. J. Biosci. Bioeng. 118, 50-55. https://doi.org/10.1016/j.jbiosc.2013.12.017 (2014).

13. Marshall, S. A. et al. Heterologous production, reconstitution and EPR spectroscopic analysis of prFMN dependent enzymes. Methods Enzymol. 620, 489-508. https://doi.org/10.1016/bs.mie.2019.03.022 (2019).

14. Richard, P., Viljanen, K. \& Penttilä, M. Overexpression of PAD1 and FDC1 results in significant cinnamic acid decarboxylase activity in Saccharomyces cerevisiae. AMB Express 5, 1-5. https://doi.org/10.1186/s13568-015-0103-x (2015).

15. Grubbe, W. S., Rasor, B. J., Krüger, A., Jewett, M. C. \& Karim, A. S. Cell-free styrene biosynthesis at high titers. Metab. Eng. 61, 89-95. https://doi.org/10.1016/j.ymben.2020.05.009 (2020).

16. Batyrova, K. A. et al. Biocatalytic in vitro and in vivo FMN prenylation and (De)carboxylase activation. ACS Chem. Biol. 15, 1874-1882. https://doi.org/10.1021/acschembio.0c00136 (2020).

17. Liu, C. et al. A systematic optimization of styrene biosynthesis in Escherichia coli BL21(DE3). Biotechnol. Biofuels 11, 1-11. https:// doi.org/10.1186/s13068-018-1017-z (2018).

18. Mukai, N., Masaki, K., Fujii, T., Kawamukai, M. \& Iefuji, H. PAD1 and FDC1 are essential for the decarboxylation of phenylacrylic acids in Saccharomyces cerevisiae. J. Biosci. Bioeng. 109, 564-569. https://doi.org/10.1016/j.jbiosc.2009.11.011 (2010). 
19. Lin, F., Ferguson, K. L., Boyer, D. R., Lin, X. N. \& Marsh, E. N. G. Isofunctional enzymes PAD1 and UbiX catalyze formation of a novel cofactor required by ferulic acid decarboxylase and 4-hydroxy-3-polyprenylbenzoic acid decarboxylase. ACS Chem. Biol. 10, 1137-1144. https://doi.org/10.1021/cb5008103 (2015).

20. Gulmezian, M., Hyman, K. R., Marbois, B. N., Clarke, C. F. \& Javor, G. T. The role of UbiX in Escherichia coli coenzyme Q biosynthesis. Arch. Biochem. Biophys. 467, 144-153. https://doi.org/10.1016/j.abb.2007.08.009 (2007).

21. Balaikaite, A. et al. Ferulic acid decarboxylase controls oxidative maturation of the prenylated flavin mononucleotide cofactor. ACS Chem. Biol. 15, 2466-2475. https://doi.org/10.1021/acschembio.0c00456 (2020).

22. Bailey, S. S. et al. The role of conserved residues in Fdc decarboxylase in prenylated flavin mononucleotide oxidative maturation, cofactor isomerization, and catalysis. J. Biol. Chem. 293, 2272-2287. https://doi.org/10.1074/jbc.RA117.000881 (2018).

23. Khusnutdinova, A. N. et al. Prenylated FMN: Biosynthesis, purification, and Fdc1 activation. Methods Enzymol. 620, 469-488. https://doi.org/10.1016/bs.mie.2019.03.021 (2019).

24. Wang, P. H. et al. Biosynthesis and activity of prenylated FMN cofactors. Cell Chem. Biol. 25, 560-570.e566. https://doi.org/10. 1016/j.chembiol.2018.02.007 (2018).

25. Annaval, T. et al. Biochemical and structural characterization of TtnD, a prenylated FMN-dependent decarboxylase from the tautomycetin biosynthetic pathway. ACS Chem. Biol. 13, 2728-2738. https://doi.org/10.1021/acschembio.8b00673 (2018).

26. Mergelsberg, M. et al. Phthaloyl-coenzyme A decarboxylase from Thauera chlorobenzoica: the prenylated flavin-, $\mathrm{K}+-$ and $\mathrm{Fe} 2+-$ dependent key enzyme of anaerobic phthalate degradation. Environ. Microbiol. 19, 3734-3744. https://doi.org/10.1111/1462-2920. 13875 (2017).

27. Payer, S. E. et al. Regioselective para-carboxylation of catechols with a prenylated flavin dependent decarboxylase. Angew Chem Int Ed 56, 13893-13897. https://doi.org/10.1002/anie.201708091 (2017).

28. Payne, K. A. P. et al. Structure and mechanism of Pseudomonas aeruginosa PA0254/HudA, a prFMN-dependent pyrrole-2-carboxylic acid decarboxylase linked to virulence. ACS Catal. 11, 2865-2878. https://doi.org/10.1021/acscatal.0c05042 (2021).

29. Payne, K. A. P. et al. Enzymatic carboxylation of 2-furoic acid yields 2,5-furandicarboxylic acid (FDCA). ACS Catal. 9, 2854-2865. https://doi.org/10.1021/acscatal.8b04862 (2019).

30. Wuensch, C. et al. Asymmetric enzymatic hydration of hydroxystyrene derivatives. Angew. Chem. Int. Ed. 52, 2293-2297. https:// doi.org/10.1002/anie.201207916 (2013).

31. Payer, S. E. et al. Exploring the catalytic promiscuity of phenolic acid decarboxylases: asymmetric, 1,6-conjugate addition of nucleophiles across 4-hydroxystyrene. Adv. Synth. Catal. 359, 2066-2075. https://doi.org/10.1002/adsc.201700247 (2017).

32. Gu, W. et al. Structural basis of enzymatic activity for the ferulic acid decarboxylase (FADase) from Enterobacter sp. Px6-4. PLOS ONE 6, 16262. https://doi.org/10.1371/journal.pone.0016262 (2011).

33. Zhang, X. et al. Biochemical characterization and substrate profiling of a reversible 2,3-dihydroxybenzoic acid decarboxylase for biocatalytic Kolbe-Schmitt reaction. Enzyme Microb. Technol. 113, 37-43. https://doi.org/10.1016/j.enzmictec.2018.02.008 (2018).

34. Bhuiya, M. W., Lee, S. G., Jez, J. M. \& Yu, O. Structure and mechanism of ferulic acid decarboxylase (FDC1) from saccharomyces cerevisiae. Appl. Environ. Microbiol. 81, 4216-4223. https://doi.org/10.1128/AEM.00762-15 (2015).

35. Lan, C. L. \& Chen, S. L. The decarboxylation of $\alpha, \beta$-unsaturated acid catalyzed by prenylated FMN-dependent ferulic acid decarboxylase and the enzyme inhibition. J. Org. Chem. 81, 9289-9295. https://doi.org/10.1021/acs.joc.6b01872 (2016).

36. Tian, G. \& Liu, Y. Mechanistic insights into the catalytic reaction of ferulic acid decarboxylase from Aspergillus niger: A QM/MM study. Phys. Chem. Chem. Phys. 19, 7733-7742. https://doi.org/10.1039/c6cp08811b (2017).

37. Mori, Y., Noda, S., Shirai, T. \& Kondo, A. Direct 1,3-butadiene biosynthesis in Escherichia coli via a tailored ferulic acid decarboxylase mutant. Nat. Commun. 12, 1-12. https://doi.org/10.1038/s41467-021-22504-6 (2021).

38. Saaret, A. et al. Directed evolution of prenylated FMN-dependent Fdc supports efficient in vivo isobutene production. Nat. Commun. 12, 1-8. https://doi.org/10.1038/s41467-021-25598-0 (2021).

39. Song, D. et al. Design, synthesis of novel 4,5-dihydroisoxazole-containing benzamide derivatives as highly potent FtsZ inhibitors capable of killing a variety of MDR Staphylococcus aureus. Bioorganic Med Chem 28, 115729. https://doi.org/10.1016/j.bmc.2020. 115729 (2020).

40. Garcia-Barrantes, P. M. \& Lindsley, C. W. Total synthesis of Gombamide A. Org. Lett. 18, 3810-3813. https://doi.org/10.1021/acs. orglett.6b01825 (2016)

41. Flores-Noria, R. et al. Synthesis and optoelectronic properties of phenylenevinylenequinoline macromolecules. New J. Chem. 38, 974-984. https://doi.org/10.1039/c3nj01193c (2014).

42. Zhao, Z., Britt, L. H. \& Murphy, G. K. Oxidative, Iodoarene-catalyzed intramolecular alkene arylation for the synthesis of polycyclic aromatic hydrocarbons. Chem. Eur. J. 24, 17002-17005. https://doi.org/10.1002/chem.201804786 (2018).

43. Gui, J. et al. Brønsted acid/visible-light-promoted Markovnikov hydroamination of vinylarenes with arylamines. Org. Biomol. Chem. 18, 956-963. https://doi.org/10.1039/c9ob02457c (2020).

44. Sharma, S., Naganaboina, R. T. \& Peddinti, R. K. Expedient synthesis of nitrovinyl substituted bicyclo[2.2.2] octenone scaffolds. RSC Adv 5, 100060-100069. https://doi.org/10.1039/c5ra20016d (2015).

45. Luo, A. Y., Bao, Y., Cheng, X. F. \& Wang, X. S. A neutral metal-free system for head-to-tail dimerization of electron-rich alkenes. Synthesis (Germany) 49, 3962-3967. https://doi.org/10.1055/s-0036-1589039 (2017).

46. Lee, Z. Y., Raghavan, S. S., Ghadessy, F. J. \& Teo, Y. N. Rapid and sensitive detection of acrylic acid using a novel fluorescence assay. RSC Adv. 4, 60216-60220. https://doi.org/10.1039/c4ra12495b (2014).

47. Shang, X. et al. Improved photoinduced fluorogenic alkene-tetrazole reaction for protein labeling. Bioconjug. Chem. 28, 2859-2864. https://doi.org/10.1021/acs.bioconjchem.7b00562 (2017).

48. Wang, Y., Song, W., Hu, W. J. \& Lin, Q. Fast alkene functionalization in vivo by photoclick chemistry: HOMO lifting of nitrile imine dipoles. Angew Chem Int Ed 48, 5330-5333. https://doi.org/10.1002/anie.200901220 (2009).

49. Song, W., Wang, Y., Qu, J. \& Lin, Q. Selective functionalization of a genetically encoded alkene-containing protein via "photoclick chemistry" in bacterial cells. J. Am. Chem. Soc. 130, 9654-9655. https://doi.org/10.1021/ja803598e (2008).

50. An, P., Lewandowski, T. M., Erbay, T. G., Liu, P. \& Lin, Q. Sterically shielded, stabilized nitrile imine for rapid bioorthogonal protein labeling in live cells. J. Am. Chem. Soc. 140, 4860-4868. https://doi.org/10.1021/jacs.8b00126 (2018).

51. Moisă, M. E. et al. Fluorescent enzyme-coupled activity assay for phenylalanine ammonia-lyases. Sci. Rep. 10, 1-11. https://doi. org/10.1038/s41598-020-75474-y (2020).

52. Markel, U. et al. A photoclick-based high-throughput screening for the directed evolution of decarboxylase OleT. Chem. Eur. J. 27, 954-958. https://doi.org/10.1002/chem.202003637 (2021).

53. Trott, O. \& Olson, A. J. AutoDock vina: Improving the speed and accuracy of docking with a new scoring function, efficient optimization, and multithreading. J. Comput. Chem. 31, 455-461 (2010).

54. Gaussian 09, Revision E.01 (Gaussian, Inc., Wallingford, CT, USA, 2009).

55. Tomasi, J., Mennucci, B. \& Cammi, R. Quantum mechanical continuum solvation models. Chem. Rev. 105, 2999-3093. https:// doi.org/10.1021/cr9904009 (2005).

56. Krieger, E. et al. Improving physical realism, stereochemistry, and side-chain accuracy in homology modeling: Four approaches that performed well in CASP8. Proteins Struct. Funct. Bioinform. 77, 114-122. https://doi.org/10.1002/prot.22570 (2009). 


\title{
Acknowledgements
}

This work was financed by the Swiss National Science Foundation (SNF) project PROMYS, grant no. IZ11Z0_166543. F.A. thanks for the financial support from the Ministry of Research, Innovation and Digitization, CNCS/CCCDI-UEFISCDI, project number PN-III-P1-1.1-PD-2019-1222/PD98 and from project: Entrepreneurship for innovation through doctoral and postdoctoral research, POCU/380/6/13/123886 co-financed by the European Social Fund, through the Operational Program for Human Capital 2014-2020. H.D. thanks the STAR-Institute of the Babeș-Bolyai University for the provided student-research fellowship.

\section{Author contributions}

H.D., A.F. contributed to the work equally. H.D. was responsible for the whole-cell biotransformation, including their HPLC-monitoring. A.F. performed the mutant library generation and initial activity screens, using the assay-plate developed together with E.Z.A.N. H.D. E.Z.A.N. performed the substrate library synthesis, while T.R. was responsible for the spectral analysis of compounds. L.C.N. performed the computational studies and was responsible for the graphical artworks. L.C.B. conceived the project and was responsible for funding together with A.F. L.C.B supervised all experiments, data and wrote the paper together with H.D., A.F., L.C.N. All authors reviewed the manuscript.

\section{Competing interests}

The authors declare no competing interests.

\section{Additional information}

Supplementary Information The online version contains supplementary material available at https://doi.org/ 10.1038/s41598-022-07110-w.

Correspondence and requests for materials should be addressed to L.C.B.

Reprints and permissions information is available at www.nature.com/reprints.

Publisher's note Springer Nature remains neutral with regard to jurisdictional claims in published maps and institutional affiliations.

\begin{abstract}
(c) (i) Open Access This article is licensed under a Creative Commons Attribution 4.0 International License, which permits use, sharing, adaptation, distribution and reproduction in any medium or format, as long as you give appropriate credit to the original author(s) and the source, provide a link to the Creative Commons licence, and indicate if changes were made. The images or other third party material in this article are included in the article's Creative Commons licence, unless indicated otherwise in a credit line to the material. If material is not included in the article's Creative Commons licence and your intended use is not permitted by statutory regulation or exceeds the permitted use, you will need to obtain permission directly from the copyright holder. To view a copy of this licence, visit http://creativecommons.org/licenses/by/4.0/.
\end{abstract}

(c) The Author(s) 2022 